\title{
Janibacter anophelis sp. nov., isolated from the midgut of Anopheles arabiensis
}

\author{
Correspondence \\ Peter Kämpfer \\ peter.kaempfer@agrar. \\ uni-giessen.de
}

\author{
Peter Kämpfer, ${ }^{1}$ Olle Terenius, ${ }^{2}$ Jenny M. Lindh ${ }^{2}$ and Ingrid Faye ${ }^{2}$ \\ ${ }^{1}$ Institut für Angewandte Mikrobiologie, Justus-Liebig-Universität Giessen, \\ IFZ-Heinrich-Buff-Ring 26-32, D-35392 Giessen, Germany \\ 2Department of Genetics, Microbiology and Toxicology, Stockholm University, S-106 91
Stockholm, Sweden
}

A Gram-positive, aerobic, non-motile strain, $\mathrm{H}_{2} \cdot 16 \mathrm{~B}^{\top}$, isolated from the midgut of the mosquito Anopheles arabiensis was investigated using a polyphasic approach. On the basis of 16S rRNA gene sequence similarity studies, strain $\mathrm{H}_{2} .16 \mathrm{~B}^{\top}$ was shown to belong to the genus Janibacter, being most closely related to Janibacter melonis (98.3\%), Janibacter terrae $(98 \cdot 5 \%)$ and Janibacter limosus (98.5\%). Chemotaxonomic data (meso-diaminopimelic acid as the diagnostic diamino acid in the cell wall and major fatty acids of iso- $\mathrm{C}_{16: 0}, \mathrm{C}_{17: 1} \omega 8 \mathrm{c}$ and $\mathrm{C}_{17: 0}$ ) supported the allocation of the strain to the genus Janibacter. The results of DNA-DNA hybridization and physiological and biochemical tests allowed the genotypic and phenotypic differentiation of strain $\mathrm{H} 2.16 \mathrm{~B}^{\top}$ from closely related species. Thus, $\mathrm{H}_{2} .16 \mathrm{~B}^{\top}$ represents a novel species of the genus Janibacter, for which the name Janibacter anophelis sp. nov. is proposed. The type strain is $\mathrm{H}_{2.16 \mathrm{~B}^{\top}}$ (= CCUG $49715^{\top}=\mathrm{CIP} 108728^{\top}$ ).
The genus Janibacter was originally proposed by Martin et al. (1997) as a member of the family Intrasporangiaceae. At present, the genus comprises three species, Janibacter limosus (Martin et al., 1997), Janibacter terrae (Yoon et al., 2000) and Janibacter melonis (Yoon et al., 2004). Janibacter brevis, originally described by Imamura et al. (2000), was shown to be a heterotypic synonym of J. terrae (Lang et al., 2003).

Strain $\mathrm{H} 2.16 \mathrm{~B}^{\mathrm{T}}$ was isolated during the characterization of organisms from the midgut of Anopheles arabiensis mosquitoes originating from Kenya (Lindh et al., 2005). Subcultivation was done on nutrient agar (Oxoid) at $30{ }^{\circ} \mathrm{C}$ for $24 \mathrm{~h}$. Gram-staining (Hucker method) was performed as described by Gerhardt et al. (1994). Cell morphology was observed under a Zeiss light microscope at $\times 1000$ using cultures that had been grown for $10 \mathrm{~h}$ or for 3 days at $30^{\circ} \mathrm{C}$ on nutrient agar. The growth ability test was performed in Luria-Bertani broth at $10-50{ }^{\circ} \mathrm{C}$ (at $5{ }^{\circ} \mathrm{C}$ intervals) with shaking at 160 r.p.m.

The 16S rRNA gene was analysed as described previously (Kämpfer et al., 2003). Phylogenetic analysis was performed by using the ARB (Ludwig et al., 2004) and MEGA version 2 (Kumar et al., 2001) software packages, after multiple alignment of the data by CLUSTAL_X (Thompson et al., 1997). Distances were obtained (using distance options according

Published online ahead of print on 7 October 2005 as DOI 10.1099/ ijs.0.63905-0.

The GenBank/EMBL/DDBJ accession number for the 16S rRNA gene sequence of Janibacter anophelis sp. nov. $\mathrm{H}_{2.16 B^{\top}}$ is AY837752. to the Kimura two-parameter model; Kumar et al., 2001) and clustering was performed using the neighbour-joining (Fig. 1) and maximum-parsimony methods by using bootstrap values based on 1000 replications. The $16 \mathrm{~S}$ rRNA gene sequence of strain $\mathrm{H} 2.16 \mathrm{~B}^{\mathrm{T}}$ was a continuous stretch of 1483 bp. Sequence similarity calculations after neighbourjoining analysis indicated that the closest relatives of strain $\mathrm{H} 2.16 \mathrm{~B}^{\mathrm{T}}$ were J. melonis $(98 \cdot 3 \%)$, J. terrae $(98 \cdot 5 \%)$ and $J$. limosus $(98 \cdot 5 \%)$. Both the neighbour-joining tree (Fig. 1) and the maximum-parsimony tree (not shown) revealed that strain $\mathrm{H} 2.16 \mathrm{~B}^{\mathrm{T}}$ clustered most closely with these species.

The results of chemotaxonomic analyses of diamino acid (as described by Kroppenstedt, 1985) and fatty acids (as described by Kämpfer \& Kroppenstedt, 1996) are given in the species description. The diagnostic diamino acid in the cellwall peptidoglycan was meso-diaminopimelic acid, essentially in accordance with the diamino acid type reported for other members of the genus (Martin et al., 1997; Yoon et al., 2000,2004 ). The fatty acids (analysed from total cell hydrolysates grown on TSBA for $24 \mathrm{~h}$ at $28{ }^{\circ} \mathrm{C}$ ) contained large amounts of iso- $\mathrm{C}_{16: 0}, \mathrm{C}_{17: 1} \omega 8 c$ and $\mathrm{C}_{17: 0}$, in addition to other fatty acids (Table 1 ). The results of the physiological characterization, performed using previously described methods (Kämpfer et al., 1991), are given in Table 2 and in the species description. DNA-DNA hybridizations between strain $\mathrm{H} 2.16 \mathrm{~B}^{\mathrm{T}}$ and the type strains of J. limosus, J. terrae and J. melonis were performed using the method described by Ziemke et al. (1998), except that for nick translation $2 \mu \mathrm{g}$ DNA was labelled during a $3 \mathrm{~h}$ incubation at $15^{\circ} \mathrm{C}$. Strain $\mathrm{H} 2.16 \mathrm{~B}^{\mathrm{T}}$ showed relatively low DNA-DNA 


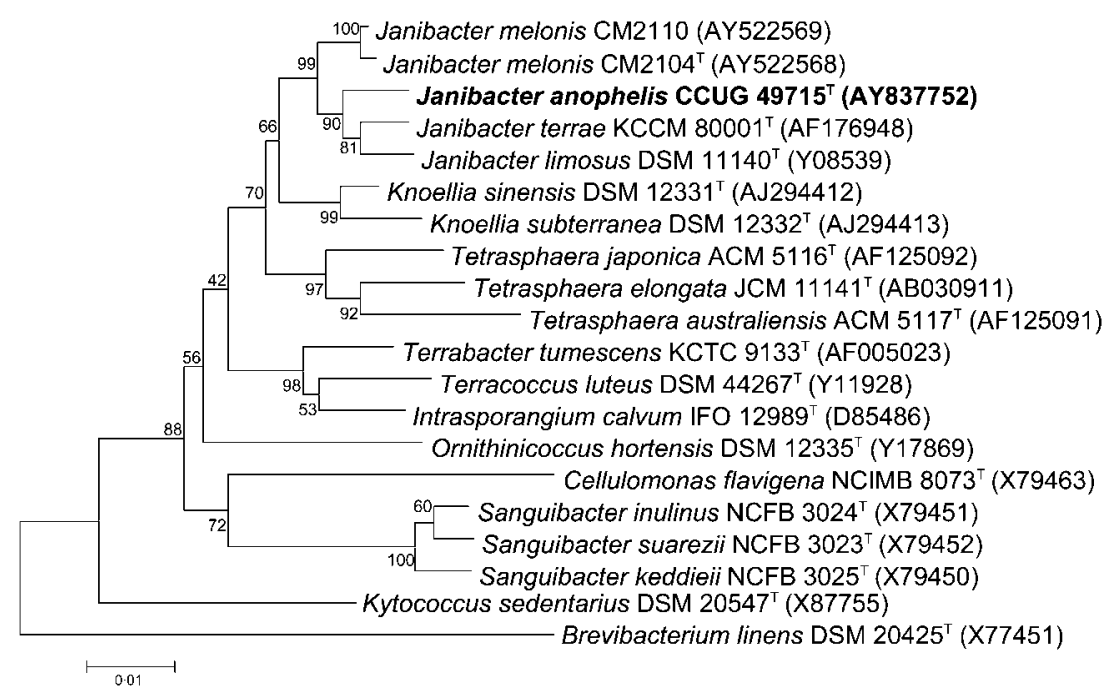

Fig. 1. Phylogenetic analysis, based on $16 \mathrm{~S}$ rRNA gene sequences available from the EMBL database (accession numbers are given in parentheses), constructed after multiple alignment of data by CLUSTAL_X (Thompson et al., 1997). Distances were obtained with distance options according to the Kimura-2 model and clustering was performed with the neighbour-joining method using the software package MEGA, version 2.1 (Kumar et al., 2001). Bootstrap values based on 1000 replications are given as percentages at branching points. Bar, 0.01 $K_{\text {nuc }}$ unit.

Table 1. Fatty acid content of Janibacter anophelis $\mathrm{H} 2.16 \mathrm{~B}^{\top}$ and other Janibacter species

Taxa: 1, Janibacter anophelis $\mathrm{H}_{2} .16 \mathrm{~B}^{\mathrm{T}} ; 2$, J. terrae DPO strains $(\mathrm{n}=10) ; 3$, J. terrae DPO $360(=\mathrm{DSM} 11214)$; 4, J. brevis DSM 13953 ${ }^{\mathrm{T}}$; 5, J. terrae DSM $13876^{\mathrm{T}}$; 6, J. limosus DSM $11140^{\mathrm{T}}$; 7, J. limosus DSM 11141; 8, J. melonis CM2104 ${ }^{\mathrm{T}}$. Data for taxa 2-7 are from Lang et al. (2003); data for J. melonis $\mathrm{CM}_{2} 104^{\mathrm{T}}$ are from Yoon et al. (2004). Values are percentages of total fatty acid content. Fatty acids $\mathrm{C}_{17: 1} \omega 6 c$, 10-methyl $\mathrm{C}_{16: 0}$ and 10-methyl $\mathrm{C}_{19: 0}$ were not present in any of the strains tested.

\begin{tabular}{|c|c|c|c|c|c|c|c|c|}
\hline Fatty acid & 1 & 2 & 3 & 4 & 5 & 6 & 7 & 8 \\
\hline $\mathrm{C}_{15: 0}$ & $3 \cdot 3$ & - & - & $0 \cdot 66$ & $0 \cdot 20$ & $0 \cdot 92$ & $0 \cdot 68$ & $12 \cdot 1$ \\
\hline $\mathrm{C}_{16: 0}$ & $2 \cdot 5$ & $0 \cdot 2-0 \cdot 9$ & $0 \cdot 2$ & $1 \cdot 55$ & $0 \cdot 55$ & $2 \cdot 12$ & $0 \cdot 92$ & $2 \cdot 4$ \\
\hline $\mathrm{C}_{17: 0}$ & $16 \cdot 4$ & $0 \cdot 8-1 \cdot 6$ & $0 \cdot 8$ & $2 \cdot 18$ & $0 \cdot 52$ & $17 \cdot 79$ & $4 \cdot 51$ & $13 \cdot 4$ \\
\hline $\mathrm{C}_{18: 0}$ & $2 \cdot 7$ & - & - & $0 \cdot 78$ & $0 \cdot 35$ & $3 \cdot 94$ & $0 \cdot 91$ & $1 \cdot 5$ \\
\hline $\mathrm{C}_{19: 0}$ & $1 \cdot 0$ & - & - & - & - & $1 \cdot 90$ & - & $0 \cdot 5$ \\
\hline $\mathrm{C}_{15: 1} \omega 6 c$ & - & $0-0 \cdot 7$ & - & $0 \cdot 36$ & $0 \cdot 28$ & - & - & $1 \cdot 4$ \\
\hline iso- $\mathrm{C}_{16: 1} \mathrm{H}$ & - & $2-3$ & $3 \cdot 07$ & $0 \cdot 54$ & $2 \cdot 08$ & - & $1 \cdot 06$ & - \\
\hline iso- $\mathrm{C}_{17: 1} \omega 9 c$ & $0 \cdot 4$ & $3-6$ & $5 \cdot 66$ & $2 \cdot 06$ & $3 \cdot 51$ & - & - & - \\
\hline anteiso- $\mathrm{C}_{17: 1} \omega 9 c$ & - & $0-1 \cdot 4$ & $1 \cdot 36$ & - & $0 \cdot 68$ & - & - & - \\
\hline $\mathrm{C}_{17: 1} \omega 8 c$ & $13 \cdot 8$ & $12-15$ & $11 \cdot 98$ & $20 \cdot 90$ & $11 \cdot 92$ & $26 \cdot 15$ & $24 \cdot 98$ & $20 \cdot 0$ \\
\hline $\mathrm{C}_{18: 1} \omega 9 c$ & $3 \cdot 9$ & $8-14$ & $8 \cdot 53$ & $15 \cdot 08$ & $13 \cdot 34$ & $17 \cdot 48$ & $11 \cdot 63$ & $7 \cdot 6$ \\
\hline $\mathrm{C}_{18: 1} \omega 7 c$ & - & - & - & $1 \cdot 25$ & $1 \cdot 28$ & - & - & - \\
\hline iso- $\mathrm{C}_{19: 1}$ & - & - & - & - & $0 \cdot 30$ & - & - & - \\
\hline iso- $\mathrm{C}_{14: 0}$ & $4 \cdot 7$ & $0 \cdot 7-1 \cdot 5$ & $1 \cdot 02$ & $0 \cdot 50$ & $1 \cdot 06$ & - & $0 \cdot 59$ & $2 \cdot 8$ \\
\hline iso- $\mathrm{C}_{15: 0}$ & $4 \cdot 4$ & $4-5$ & $4 \cdot 43$ & $3 \cdot 72$ & $2 \cdot 38$ & $0 \cdot 56$ & - & $5 \cdot 1$ \\
\hline anteiso- $\mathrm{C}_{15: 0}$ & $0 \cdot 6$ & $0-0 \cdot 6$ & $0 \cdot 55$ & - & $0 \cdot 44$ & - & - & $1 \cdot 2$ \\
\hline iso- $\mathrm{C}_{16: 0}$ & $32 \cdot 9$ & $36-44$ & $41 \cdot 07$ & $30 \cdot 45$ & $38 \cdot 10$ & $12 \cdot 21$ & $34 \cdot 82$ & $16 \cdot 7$ \\
\hline iso- $\mathrm{C}_{17: 0}$ & $3 \cdot 3$ & $4-5$ & $4 \cdot 17$ & $6 \cdot 56$ & $3 \cdot 86$ & $1 \cdot 47$ & $0 \cdot 73$ & $4 \cdot 1$ \\
\hline anteiso- $\mathrm{C}_{17: 0}$ & $2 \cdot 0$ & $3-6$ & $5 \cdot 89$ & $1 \cdot 97$ & $5 \cdot 67$ & - & - & $2 \cdot 2$ \\
\hline 10-methyl $C_{17: 0}$ & $2 \cdot 1$ & $4-5$ & $5 \cdot 16$ & $2 \cdot 68$ & $5 \cdot 97$ & $2 \cdot 84$ & $5 \cdot 97$ & $0 \cdot 4$ \\
\hline iso- $\mathrm{C}_{18: 0}$ & $3 \cdot 2$ & $0-0 \cdot 8$ & $0 \cdot 57$ & $1 \cdot 25$ & $0 \cdot 92$ & $3 \cdot 36$ & $3 \cdot 48$ & $1 \cdot 1$ \\
\hline 10 -methyl $C_{18: 0}$ & - & - & - & - & $0 \cdot 20$ & $0 \cdot 78$ & - & $0 \cdot 3$ \\
\hline Feature $4^{\star}$ & $1 \cdot 2$ & $3-5$ & $2 \cdot 75$ & $3 \cdot 85$ & $3 \cdot 85$ & $1 \cdot 08$ & $2 \cdot 41$ & $2 \cdot 1$ \\
\hline Feature $6^{*}$ & - & - & - & $3 \cdot 66$ & $2 \cdot 32$ & - & - & - \\
\hline Feature $7^{*}$ & - & $0-1 \cdot 6$ & $1 \cdot 36$ & - & - & - & - & - \\
\hline Feature $8^{*}$ & $0 \cdot 9$ & $1 \cdot 5-2 \cdot 3$ & $1 \cdot 60$ & - & - & $7 \cdot 40$ & $4 \cdot 96$ & $3 \cdot 7$ \\
\hline
\end{tabular}

${ }^{\star}$ Unknown fatty acids; these compounds have no names listed in the peak library file of the MIDI system and therefore were not identified or included in the percentage calculation. 
Table 2. Biochemical properties of Janibacter anophelis $\mathrm{H} 2.16 \mathrm{~B}^{\top}$ and the type strains of other Janibacter species

Taxa: 1, Janibacter anophelis $\mathrm{H} 2.16 \mathrm{~B}^{\mathrm{T}} ; 2$, J. terrae DSM $13876^{\mathrm{T}} ; 3$, J. limosus DSM $11140^{\mathrm{T}}$; 4, J. melonis CM $2104^{\mathrm{T}}$. All data are from this study. W, Weak reaction; D, delayed reaction.

\begin{tabular}{|lcccc|}
\hline Characteristic & $\mathbf{1}$ & $\mathbf{2}$ & $\mathbf{3}$ & $\mathbf{4}$ \\
\hline Growth at $37^{\circ} \mathrm{C}$ (on TSBA) & + & $\mathrm{W}$ & - & + \\
Utilization of: & & & & \\
Acetate & + & + & - & + \\
N-Acetyl-D-glucosamine & - & $\mathrm{D}$ & $\mathrm{D}$ & $\mathrm{W}$ \\
cis-Aconitate & - & + & - & - \\
Adipate & - & + & - & $\mathrm{W}$ \\
4-Aminobutyrate & $\mathrm{W}$ & - & - & + \\
L-Azelate & - & $\mathrm{W}$ & - & - \\
D-Fructose & - & $+^{*}$ & + & - \\
D-Gluconate & + & $+^{*}$ & + & - \\
D-Glucose & + & $+^{*}$ & + & $\mathrm{W}$ \\
i-Inositol & + & $+^{*}$ & $\mathrm{~W}$ & - \\
D-Maltose & + & $\mathrm{D}^{*}$ & + & $\mathrm{W}$ \\
D-Mannose & + & + & $\mathrm{W}$ & $\mathrm{W}$ \\
2-Oxoglutarate & - & + & - & + \\
L-Proline & $\mathrm{W}$ & $+^{*}$ & $\mathrm{~W}$ & + \\
Putrescine & - & $+^{*}$ & + & - \\
L-Suberate & - & + & $\mathrm{W}$ & $\mathrm{W}$ \\
Sucrose & + & $+^{*}$ & + & $\mathrm{W}$ \\
D-Trehalose & + & $\mathrm{D}^{*}$ & + & + \\
& & & & \\
\hline
\end{tabular}

${ }^{*}$ Reaction also reported for J. terrae strain DPO 1361 (=DSM 11220) and J. brevis DSM $13953^{\mathrm{T}}$ [a later synonym of J. terrae (Lang et al., 2003)].

relatedness to J. melonis DSM $16063^{\mathrm{T}}(12 \cdot 0 \%$; reciprocal reaction, $9 \cdot 8 \%)$, J. terrae DSM $13876^{\mathrm{T}}(13.7 \%$; reciprocal reaction, $32 \cdot 4 \%)$ and $J$. limosus DSM $11140^{\mathrm{T}}(34 \cdot 0 \%$; reciprocal reaction, $19 \cdot 2 \%)$. For these reasons, it is clear that strain $\mathrm{H} 2.16 \mathrm{~B}^{\mathrm{T}}$ represents a novel species of the genus Janibacter, for which we propose the name Janibacter anophelis sp. nov.

\section{Description of Janibacter anophelis sp. nov.}

Janibacter anophelis (a.no'phe.lis. N.L. gen. n. anophelis from a mosquito of the genus Anopheles, as the type strain was isolated from the midgut of Anopheles arabiensis).

Gram-positive and oxidase-positive; shows oxidative metabolism. Non-motile, non-endospore-forming cocci of $1 \cdot 0$ $1.5 \mu \mathrm{m}$ in diameter. Very young cultures show rod-like cells. Good growth (visible colonies with a diameter $>1 \mathrm{~mm}$ ) occurs after 3 days incubation on nutrient agar at $25-30{ }^{\circ} \mathrm{C}$. Grows in Luria-Bertani broth at $20-40^{\circ} \mathrm{C}$, with an optimum at $35^{\circ} \mathrm{C}$. Generation time at $35^{\circ} \mathrm{C}$ is $44 \mathrm{~min}$. mesoDiaminopimelic acid is the diagnostic diamino acid in the cell-wall peptidoglycan. The fatty acid content is mainly iso$\mathrm{C}_{16: 0}, \mathrm{C}_{17: 1} \omega 8 c$ and $\mathrm{C}_{17: 0}$. Different 10-methyl acids are present. Results of carbon source utilization tests (including differentiating characteristics) are shown in Table 2. The type strain does not produce acids from the following sugars: glucose, lactose, sucrose, D-mannitol, dulcitol, salicin, adonitol, inositol, sorbitol, L-arabinose, raffinose, L-rhamnose, maltose, D-xylose, trehalose, cellobiose, methyl D-glucoside, erythritol, melibiose or arabitol. The following carbon sources are utilized as a sole source of carbon (after $48 \mathrm{~h}$ incubation): D-gluconate, D-glucose, D-maltose, Dmannose, sucrose, D-trehalose, i-inositol, acetate, propionate, 4-aminobutyrate, fumarate, glutarate, 3-hyroxybutyrate, DL-lactate, L-malate, pyruvate, L-alanine, L-aspartate, Lhistidine, L-leucine, L-proline and L-serine. The following carbon sources are not utilized: $N$-acetyl-D-galactosamine, $\mathrm{N}$-acetyl-D-glucosamine, L-arabinose, $p$-arbutin, D-cellobiose, D-fructose, D-galactose, melibiose, L-rhamnose, D-ribose, salicin, D-xylose, adonitol, maltitol, D-mannitol, sorbitol, putrescine, cis-aconitate, trans-aconitate, adipate, L-azelate, citrate, itaconate, mesaconate, 2-oxoglutarate, L-suberate, $\beta$ alanine, L-ornithine, L-phenylalanine, L-tryptophan, DL-3hydroxybenzoate, DL-4-hydroxybenzoate or L-phenylacetate.

The type strain, $\mathrm{H} 2.16 \mathrm{~B}^{\mathrm{T}}\left(=\mathrm{CCUG} 49715^{\mathrm{T}}=\mathrm{CIP} 108728^{\mathrm{T}}\right.$ ), was isolated from the midgut of Anopheles arabiensis from Kenya.

\section{Acknowledgements}

We thank Jean Euzéby for his help with the etymology of the species epithet.

\section{References}

Gerhardt, P., Murray, R. G. E., Wood, W. A. \& Krieg, N. R. (editors) (1994). Methods for General and Molecular Bacteriology. Washington, DC: American Society for Microbiology.

Imamura, Y., Ikeda, M., Yoshida, S. \& Kuraishi, H. (2000). Janibacter brevis sp. nov., a new trichloroethylene-degrading bacterium isolated from polluted environments. Int J Syst Evol Microbiol 50, 1899-1903.

Kämpfer, P. \& Kroppenstedt, R. M. (1996). Numerical analysis of fatty acid patterns of coryneform bacteria and related taxa. Can J Microbiol 42, 989-1005.

Kämpfer, P., Steiof, M. \& Dott, W. (1991). Microbiological characterisation of a fuel-oil contaminated site including numerical identification of heterotrophic water and soil bacteria. Microb Ecol 21, 227-251.

Kämpfer, P., Dreyer, U., Neef, A., Dott, W. \& Busse, H.-J. (2003). Chryseobacterium defluvii sp. nov., isolated from wastewater. Int J Syst Evol Microbiol 53, 93-97.

Kroppenstedt, R. M. (1985). Fatty acid menaquinone analysis of actinomycetes and related organisms. In Chemical Methods in Bacterial Systematics, pp. 173-199. Edited by M. Goodfellow \& D. E. Minnikin. London: Academic Press.

Kumar, S., Tamura, K., Jakobsen, I. B. \& Nei, M. (2001). MEGA2: molecular evolutionary genetics analysis software. Bioinformatics $\mathbf{1 7}$, 1244-1245.

Lang, E., Kroppenstedt, R. M., Swiderski, J., Schumann, P., Ludwig, W., Schmid, A. \& Weiss, N. (2003). Emended description of Janibacter terrae, including ten dibenzofuran-degrading strains and Janibacter brevis as its later heterotypic synonym. Int J Syst Evol Microbiol 53, 1999-2005. 
Lindh, J. M., Terenius, O. \& Faye, I. (2005). 16S rRNA gene-based identification of midgut bacteria from field-caught Anopheles gambiae sensu lato and $A$. funestus mosquitoes reveals new species related to known insect symbionts. Appl Environ Microbiol 71, 7217-7223.

Ludwig, W., Strunk, O., Westram, R. \& 29 other authors (2004). ARB: a software environment for sequence data. Nucleic Acids Res 32, 1363-1371.

Martin, K., Schumann, P., Rainey, F. A., Schuetze, B. \& Groth, I. (1997). Janibacter limosus gen. nov., sp. nov., a new actinomycete with mesodiaminopimelic acid in the cell wall. Int J Syst Bacteriol 47, 529-534.

Thompson, J. D., Gibson, T. J., Plewniak, F., Jeanmougin, F. \& Higgins, D. G. (1997). The CLUSTAL_X windows interface: flexible strategies for multiple sequence alignment aided by quality analysis tools. Nucleic Acids Res 25, 4876-4882.

Yoon, J.-H., Lee, K.-C., Kang, S.-S., Kho, Y. H., Kang, K. H. \& Park, Y.-H. (2000). Janibacter terrae sp. nov., a bacterium isolated from soil around a wastewater treatment plant. Int J Syst Evol Microbiol 50, 1821-1827.

Yoon, J.-H., Lee, B. H., Yeo, S.-H. \& Choi, J.-E. (2004). Janibacter melonis sp. nov., isolated from abnormally spoiled oriental melon in Korea. Int J Syst Evol Microbiol 54, 1975-1980.

Ziemke, F., Höfle, M. G., Lalucat, J. \& Rosselló-Mora, R. (1998). Reclassification of Shewanella putrefaciens Owen's genomic group II as Shewanella baltica sp. nov. Int J Syst Bacteriol 48, 179-186. 